\title{
Burden and treatment patterns of invasive fungal infections in hospitalized patients in the Middle East: real-world data from Saudi Arabia and Lebanon
}

\author{
Adel F Alothman' \\ Abdulhakeem $\bigcirc$ Althaqafi ${ }^{2}$ \\ Madonna J Matar ${ }^{3}$ \\ Rima Moghnieh ${ }^{4}$ \\ Thamer H Alenazi' \\ Fayssal M Farahat ${ }^{2}$ \\ Shelby Corman ${ }^{5}$ \\ Caitlyn T Solem ${ }^{5}$ \\ Nirvana Raghubir ${ }^{6}$ \\ Cynthia Macahilig ${ }^{7}$ \\ Claudie Charbonneau ${ }^{8}$ \\ Jennifer M Stephens ${ }^{5}$ \\ 'College of Medicine, King Abdullah \\ International Medical Research \\ Center, King Saud bin Abdulaziz \\ University for Health Sciences, Riyadh, \\ Saudi Arabia; ${ }^{2}$ Department of Infection \\ Prevention and Control, King Abdullah \\ International Medical Research \\ Center, King Saud bin AbdulAziz \\ University for Health Sciences, King \\ AbdulAziz Medical City, Jeddah, Saudi \\ Arabia; ${ }^{3}$ Department of Infectious \\ Disease, Notre Dame de Secours \\ University Hospital, Byblos, Lebanon; \\ ${ }^{4}$ Department of Internal Medicine, \\ Makassed General Hospital, Beirut, \\ Lebanon; ${ }^{5}$ Real-world Evidence/ \\ Data Analytics Center of Excellence, \\ Pharmerit International, Bethesda, MD, \\ USA; ${ }^{6}$ Pfizer, New York, NY, ${ }^{7}$ Medical \\ Data Analytics, Parsippany, NJ, USA; \\ ${ }^{8}$ Pfizer International Operation, Paris, \\ France
}

Correspondence: Jennifer M Stephens Pharmerit International, 4350 East West Highway, Suite 430, Bethesda, MD 208I4, USA

Tel $+|24082| 1290$

$\mathrm{Fax}+\mid 2408211296$

Email jstephens@pharmerit.com
This article was published in the following Dove Press journal:

Infection and Drug Resistance

2 February 2017

Number of times this article has been viewed

Objectives: The objective of this study was to document the burden and treatment patterns associated with invasive fungal infections (IFIs) due to Candida and Aspergillus species in Saudi Arabia and Lebanon.

Methods: A retrospective chart review study was conducted using data recorded from 2011 to 2012 from hospitals in Saudi Arabia and Lebanon. Patients were included if they had been discharged with a diagnosis of IFI due to Candida or Aspergillus, which was culture proven or suspected based on clinical criteria. Hospital data were abstracted for a random sample of patients to capture demographics, treatment patterns, hospital resource utilization, and clinical outcomes. Descriptive results were reported.

Results: Five hospitals participated and provided data on 102 patients with IFI (51 from Lebanon and 51 from Saudi Arabia). The mean age of the patients was 55 years, and 55\% were males. Comorbidities included diabetes (41\%), coronary artery disease (24\%), leukemia (19\%), moderate-to-severe renal disease (16\%), congestive heart failure (15\%), and chronic obstructive pulmonary disease $(15 \%)$. Twenty percent of patients received corticosteroids prior to admission and $26 \%$ had received chemotherapy in the past 90 days. Inpatient mortality was $42 \%$, and the mean hospital length of stay was $32.4 \pm 28.6$ days. Fifty-five percent of patients required intensive care unit admission (17.2 \pm 14.1 days), 37\% required mechanical ventilation (13.7 \pm 13.2 days), and $11 \%$ required dialysis (14.6 \pm 14.2 days). The most commonly used firstline antifungal was fluconazole.

Conclusion: Patients with IFI in Saudi Arabia and Lebanon frequently have multiple medical comorbidities and may not have traditionally observed IFI risk factors. Efforts to increase use of rapid diagnostic tests and appropriate antifungal treatments may impact the substantial mortality and high length of stay observed in these patients.

Keywords: Candida, Aspergillus, length of stay, resource use, antifungal, mortality

\section{Introduction}

The incidence and risk of invasive fungal infections (IFIs) continue to rise. The epidemiology of IFI has changed over time and is thought to be affected by patient factors, environmental considerations, and antifungal exposure. What was once a disease primarily caused by Candida albicans, IFIs caused by both C. albicans and invasive molds, predominantly Aspergillus species, have been increasing. ${ }^{1,2}$ These changes have driven the need for better methods of organism and species identification.

IFIs are insidious. Infections have often become well established by the time positive diagnostic evidence is found. The complexity of these infections necessitates rapid diagnosis and treatment. In recent years, the identification of rapid diagnostic methods 
and new antifungal agents has advanced the armamentarium for IFI management. Despite these improvements, consensus regarding how best to utilize these diagnostic methods and the optimal time to start antifungal treatment is still evolving.

In the Middle East, there is limited information available about the real-world burden of IFI, including epidemiology, mortality, treatment patterns, and resource utilization associated with these infections. The objectives of this study were therefore to document the microbiologic profile of IFI in Lebanon and Saudi Arabia and to describe the antifungal treatment received by these patients, in terms of drug selection and appropriateness, and to document hospital resource utilization and clinical outcomes among patients with these infections.

\section{Methods Study design}

It was the responsibility of the study investigators (SIs) to have approval of the study from the local IRB/ethics committee. All sites submitted this study at their local institutions and received approval or exemption; no centralized ethics approval was sought for this study. In all cases, informed consent was not required for this non-interventional medical chart review study. This study is a retrospective, observational medical chart review that took place at five study sites in Saudi Arabia (two sites) and Lebanon (three sites). The purpose of this study is to describe the epidemiology, mortality, treatment patterns, and resource utilization associated with IFI for each country. Note that this analysis is descriptive of each country, not comparative between countries or sites.

\section{Inclusion and exclusion criteria}

In order to be included, patients hospitalized between January 1, 2011, and December 31, 2012, had to have suspected or culture-proven IFI due to Candida or Aspergillus, must have received definitive antifungal drug therapy, and must have met at least one of the following additional criteria: surgical and/or medical patients at risk for IFI in the intensive care unit (ICU; stay must be $\geq 3$ days in duration), patients with cancer and/or bone marrow transplant or stem cell transplant, patients who had undergone a solid organ transplant, and immunocompromised patients, including (but not limited to) those positive for HIV, diabetic patients, or those taking corticosteroids. These criteria aimed to include both patients with more typically observed and identified IFI risk factors (cancer, bone marrow/stem cell transplant, solid organ transplant) and patients meeting a broader definition for IFI risk, including those with an ICU admission $\geq 3$ days.

\section{Patient characteristics, treatment patterns, and outcomes}

Basic patient demographics were collected from the medical charts, including age, sex, weight, comorbid conditions, history of chemotherapy, and prior use of corticosteroids. Microbiologic identification, site of IFI confirmatory culture, and antifungal sensitivities were collected as reported by the abstracting physician. Antifungal therapy treatment patterns were collected in detail to include drug, dose, route, timing (days to start antifungal treatment), and changes in therapy.

Data collection for patterns of health care resource use included the following: pathological tests, microbiological/ virological tests, laboratory tests, radiological/imaging tests, procedures such as days of mechanical ventilation and dialysis/renal replacement therapy, and length of hospital stay, overall and by location (emergency department, ICU, regular ward, intermediate care/high dependency unit).

Clinical outcomes collected included overall in-hospital mortality (IFI related and non-IFI related), relapse and/or rehospitalization for IFI or rehospitalization for any reason within 30 days post discharge, and clinical response of IFI cases at the time of discharge (clinical cure, improvement, failure, indeterminate).

\section{Statistical analysis}

Binary and categorical end points were summarized in terms of percentages in each category, and continuous variables were presented using number of observations (when less than the entire sample), arithmetic mean, and standard deviation. As a descriptive study for each country, no $P$-values are provided for between-country or between-site comparisons.

\section{Results}

\section{Patient-level demographic and clinical characteristics}

Detailed medical chart data were collected for 102 patients across the five study sites, 51 from Saudi Arabia and 51 from Lebanon. The majority of the patients were indicated by the study investigator to be immunocompromised (81\%; Table 1). Other common reasons for inclusion were ICU admission $\geq 3$ days $(51 \%)$ and cancer and/or bone marrow transplant or stem cell transplant (45\%). Only two patients $(2 \%)$ were solid organ transplant recipients.

Almost all patients $(92 \%)$ had at least one comorbidity (Table 1). The mean number of comorbid conditions per patient was 2.4 , with the most common conditions being diabetes, coronary artery disease, and leukemia. A small proportion of 
Table I Patient profile - IFI

\begin{tabular}{|c|c|c|c|}
\hline & $\begin{array}{l}\text { Overall } \\
(\mathrm{N}=102)\end{array}$ & $\begin{array}{l}\text { Saudi Arabia } \\
(n=5 I)\end{array}$ & $\begin{array}{l}\text { Lebanon } \\
(n=5 I)\end{array}$ \\
\hline Male, \% & 55 & 55 & 55 \\
\hline Age at hospital admission, mean \pm SD & $55.2 \pm 25.1$ & $56.8 \pm 25.5$ & $53.6 \pm 25.0$ \\
\hline Any comorbidities, $\%$ & 92 & 92 & 92 \\
\hline Number of reported comorbidities, mean \pm SD & $2.4 \pm 1.7$ & $2.4 \pm 1.7$ & $2.4 \pm 1.7$ \\
\hline Diabetes, \% & 41 & 55 & 27 \\
\hline Coronary artery disease, $\%$ & 24 & 12 & 35 \\
\hline Leukemia, \% & 19 & 10 & 27 \\
\hline Moderate-to-severe renal disease, $\%$ & 16 & 14 & 18 \\
\hline Congestive heart failure, $\%$ & 15 & 16 & 14 \\
\hline Chronic pulmonary disease, $\%$ & 15 & 12 & 18 \\
\hline Cerebrovascular disease, $\%$ & 14 & 20 & 8 \\
\hline Hypertension, \% & 9 & 14 & 4 \\
\hline \multicolumn{4}{|l|}{ Criteria met to be included in study, $\%$} \\
\hline Surgical and/or medical patient at risk for IFI in the ICU & 51 & 47 & 55 \\
\hline $\begin{array}{l}\text { Has/had cancer and/or is a bone marrow or stem cell } \\
\text { transplant patient }\end{array}$ & 45 & 31 & 59 \\
\hline Had undergone a solid organ transplant & 2 & 2 & 2 \\
\hline Immunocompromised & 81 & 76 & 86 \\
\hline \multicolumn{4}{|l|}{ Drug use prior to admission } \\
\hline Antifungals prior to admission, $\%$ & 10 & 0 & 20 \\
\hline Corticosteroids prior to admission, $\%$ & 20 & 6 & 33 \\
\hline Chemotherapy within 90 days prior to admission, $\%$ & 26 & 6 & 47 \\
\hline \multicolumn{4}{|l|}{ Days from hospital admission to diagnosis, $\%$} \\
\hline Within 2 days of admission & 34 & 53 & 16 \\
\hline After 2 days of admission & 66 & 47 & 84 \\
\hline \multicolumn{4}{|l|}{ ANC } \\
\hline Patients with valid information, $\mathrm{n}$ & 27 & 3 & 24 \\
\hline Neutropenic (ANC $<500$ cells $/ \mu \mathrm{L}$ ), \% & II & 0 & 13 \\
\hline
\end{tabular}

Note: ${ }^{a} C U$ stay must be at least 72 hours in duration.

Abbreviations: IFI, invasive fungal infection; ICU, intensive care unit; ANC, absolute neutrophil count.

patients were receiving antifungals $(10 \%)$ or corticosteroids (20\%) prior to admission, and $26 \%$ of patients had received chemotherapy within the 90 days prior to admission.

Of the 102 IFI patients, $100(98 \%)$ had culture results available, and of these, $78(78 \%)$ had at least one positive culture. Patients most frequently had positive cultures collected from blood (44\%), pulmonary sites (33\%), and urine (28\%). Figure 1 shows the microbiologic profile of available Candida and Aspergillus cultures. For Candida infections ( $\mathrm{n}=70$ cultures), 56\% were C. albicans, 20\% were Candida tropicalis, 14\% were Candida glabrata, and 13\% were another type of Candida. For Aspergillus cultures ( $\mathrm{n}=10), 60 \%$ were Aspergillus fumigatus, 10\% were Aspergillus niger, 10\% were Aspergillus flavus, and $20 \%$ were unknown. Two patients (2\%) had positive cultures for both Candida and Aspergillus. Aspergillus galactomannan antigen testing was performed in $11 \%$ of patients.

\section{Antifungal treatment patterns}

Table 2 shows the susceptibility profile as reported by the abstracting physician for the 29 Candida specimens tested for antifungal susceptibility. All susceptibility results were reported in patients from Saudi Arabia; no testing was performed in Lebanon or in Aspergillus isolates from either country. Only $62 \%$ and $35 \%$ of specimens were sensitive to fluconazole and itraconazole, respectively. While some specimens were sensitive to flucytosine and itraconazole (Table 2), these agents ultimately were not prescribed to any of the patients included in this study.

The median time from admission to diagnosis was 6 days (range 0 to 92 days), and active therapy was initiated at a median of 1 day thereafter (range 0 to 93 days post admission). Among the 62 patients who had both a culture collected and active therapy initiated, a median of 2.5 days (range -3 to 80 days) passed between these events.

Overall, 78 patients $(76 \%)$ received at least one antifungal considered to be active against the infecting fungal species. The 102 IFI patients were prescribed a total of 111 first-line drugs, with nearly two-thirds including fluconazole (Figure 2). Forty-two patients (41\%) required a total of 43 second-line drugs, with $26 \%$ of patients discontinuing the 
A

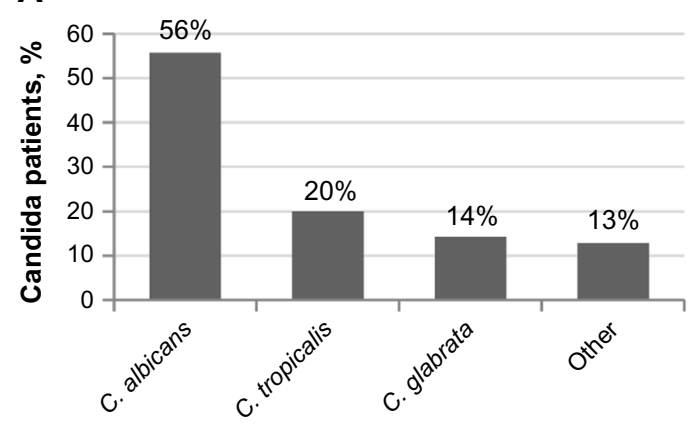

B

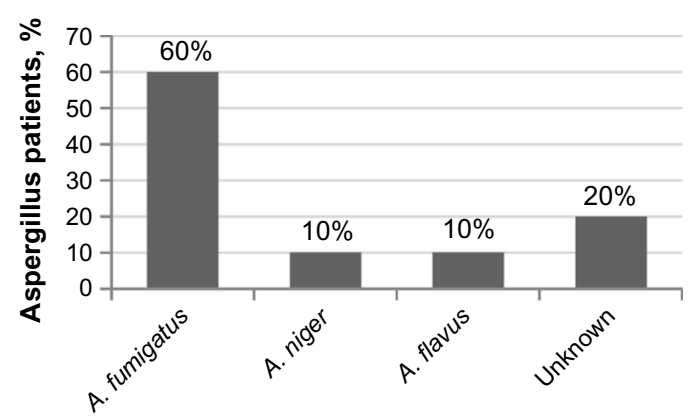

Figure I Microbiologic profile of invasive fungal infections.

Notes: (A) Candida ( $\mathrm{n}=70$ cultures). $\mathrm{n}=2$ patients cultured both Candida albicans and Candida glabrata. Other Candida included C. parapsilosis ( $\mathrm{n}=3$ ), $C$. famata ( $\mathrm{n}=2$ ), C. krusei $(n=1)$, C. lusitaniae $(n=1)$, C. rugosa $(n=1)$, and non-albicans (otherwise unspecified, $n=1)$. (B) Aspergillus ( $n=10$ cultures).

Abbreviations: C. albicans, Candida albicans; C. glabrata, Candida glabrata; C. parapsilosis, Candida parapsilosis; C. famata, Candida famata; C. krusei, Candida krusei; C. lusitaniae, Candida lusitaniae; C. rugosa, Candida rugosa; C. tropicalis, Candida tropicalis; A. fumigatus, Aspergillus fumigatus; A. niger, Aspergillus niger; A. flavus, Aspergillus flavus.

first-line regimen due to lack of response. The most commonly selected second-line antifungals were voriconazole $(35 \%)$ and caspofungin $(30 \%)$. Eleven patients $(11 \%)$ required a total of 13 third-line drugs, with the most common being amphotericin B (36\%).

\section{Health care resource utilization}

Table 3 highlights key health care resource utilization across both countries. Patients with IFI stayed in the hospital for a mean of 32 days and spent the majority of that time in general wards (19 days across all patients), while $55 \%$ of patients required admission to an ICU. Thirty-eight patients with IFI $(37 \%)$ required mechanical ventilation, with a mean duration of 13.7 days per patient. In addition, eleven patients $(11 \%)$ required hemodialysis for a mean of 14.6 days.

\section{Clinical outcomes}

Table 4 shows the clinical outcomes with $42 \%$ of patients dying in the hospital; however, the cause of death included IFI in only $33 \%$. Few patients relapsed or were rehospitalized for IFI ( $2 \%$ for both).

Table 2 Invasive fungal infection susceptibility profile ${ }^{a}$

\begin{tabular}{lll}
\hline Antifungal & N tested & \% sensitive \\
\hline Caspofungin & 8 & 100 \\
Amphotericin B & 28 & 96 \\
Voriconazole & 19 & 79 \\
Flucytosine & 21 & 71 \\
Fluconazole & 29 & 62 \\
Itraconazole & 17 & 35 \\
\hline
\end{tabular}

Notes: aSusceptibility as reported by the abstracting physician. No specific minimum inhibitory concentration-fold parameters were provided to abstracting physicians; this was based on their clinical judgment.

\section{Discussion}

This is the first real-world observational study of IFI in the Middle East that comprehensively assessed the mortality, treatment patterns, and resource use burden. Our results indicate that IFI represents a substantial burden to hospitals in the Middle East, with significant unmet needs, including delays in time to active therapy initiation, low utilization of rapid diagnostic tests, and suboptimal antifungal treatment, all of which may have impacted the high mortality rates and extended length of hospital stay seen in this population.

In patients with IFI, difficulty in recognizing the infection as fungal may lead to delays in initiation of antifungal therapy. In this study, the median time from admission to diagnosis was 6 days, and antifungal therapy was delayed by an additional day. Previous studies have shown a clear relationship between time to initiation of antifungal therapy and in-hospital mortality, with delays as short as 12 hours associated with increased risk of mortality. ${ }^{3}$ Despite the long delays to antifungal therapy in this study, in-hospital mortality was similar to that seen in previous studies. ${ }^{3-6}$

Over two-thirds of patients with IFI received fluconazole as part of their first-line antifungal regimen, which is likely due to its historical position as a first-line antifungal in clinical practice guidelines and low cost. However, antifungal susceptibility testing revealed that only $62 \%$ of the tested Candida isolates were sensitive to fluconazole and $42 \%$ of patients were switched to a second antifungal regimen. The use of initial antifungal regimens that are not active against the infecting organism can lead to further delays in the initiation of appropriate antifungal therapy, which could further worsen clinical outcomes. Recent guidelines in the Middle 


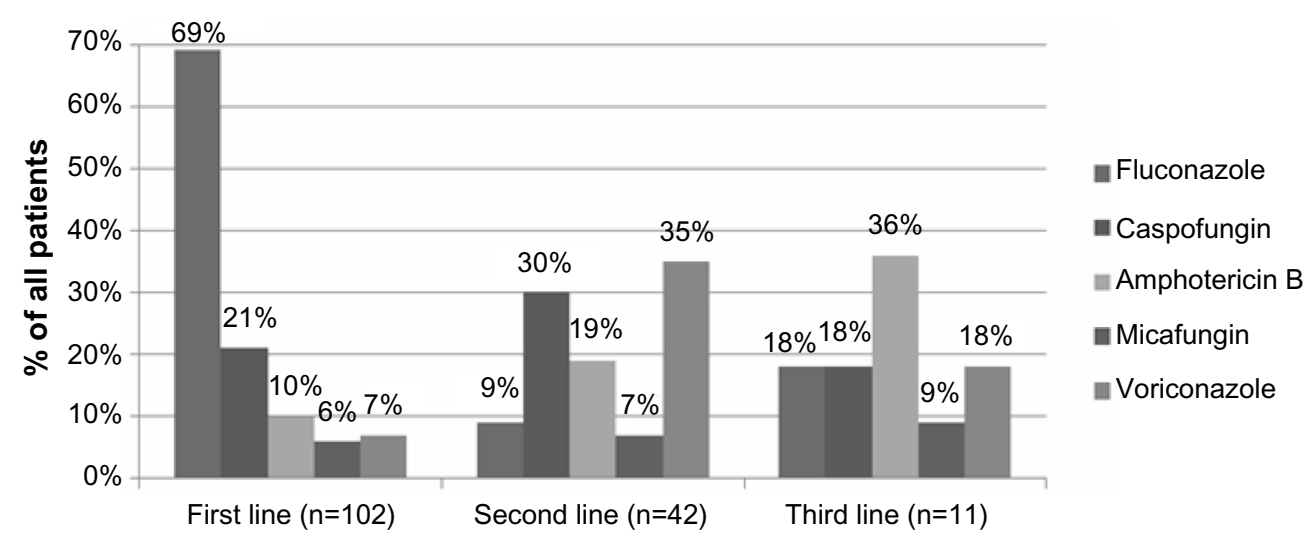

Figure 2 Medications utilized for invasive fungal infection treatment.

East recommend the echinocandins as the first-line agents for Candida, given emerging resistance to fluconazole. ${ }^{2}$

IFIs have a significant impact on the morbidity and mortality of immunocompromised patients and are also known to affect nontypical hosts such as patients with medical comorbidities and those in the ICU. ${ }^{7,8} \mathrm{~A}$ combination of typical (cancer, bone marrow/stem cell transplant, solid organ transplant) and nontypical (ICU admission $\geq 3$ days) risk factors were used in the selection of patients for this study; however, less than half of the study patients had at least one typical risk factor. The findings in this study highlight the trends in other regions that nontypical hosts with comorbidities such as cardiovascular, pulmonary, and renal diseases represent key patient populations at risk for developing IFI ${ }^{8,9}$ Earlier consideration for the potential of IFI in medically comorbid patients may reduce the time to antifungal treatment and improve outcomes.

The economics of IFI are driven by the length of hospitalization including ICU, general ward stay and the total length of stay. Antifungal treatment and cost are dependent on the antifungal chosen, weight of the patient for weight-based therapies, and duration of therapy. In this study, by simply documenting the current resource use burden for hospitals managing IFI patients and the suboptimal application of rapid diagnostic testing and antifungal treatment (despite current guidelines in the Middle East for IFI), the hope is that improvements in care may be achieved. ${ }^{1,2}$ The costs

Table 3 IFI health care resource utilization

\begin{tabular}{|c|c|c|c|}
\hline & $\begin{array}{l}\text { Total } \\
(\mathrm{N}=102)\end{array}$ & $\begin{array}{l}\text { Saudi Arabia } \\
(n=5 I)\end{array}$ & $\begin{array}{l}\text { Lebanon } \\
(n=5 I)\end{array}$ \\
\hline Total LOS (days), mean \pm SD & $32.4 \pm 28.6$ & $39.0 \pm 35.9$ & $25.9 \pm 16.7$ \\
\hline $\mathrm{ER}, \mathrm{n}(\%)$ & $7(6.9 \%)$ & 7 (I3.7\%) & $0(0.0 \%)$ \\
\hline Days spent in $E R$, mean $\pm S D$ & $3.3 \pm 0.5$ & $3.3 \pm 0.5$ & - \\
\hline General ward, n (\%) & $66(64.7 \%)$ & $44(86.3 \%)$ & $22(43.1 \%)$ \\
\hline Days spent in general ward, mean \pm SD & $29.4 \pm 32.4$ & $32.0 \pm 36.5$ & $24.2 \pm 22.1$ \\
\hline HDU/Intermediate, n (\%) & $25(24.5 \%)$ & $0(0 \%)$ & $25(49 \%)$ \\
\hline Days spent in HDU/intermediate, mean $\pm S D$ & $15.3 \pm 10.1$ & - & $15.3 \pm 10.1$ \\
\hline ICU, n (\%) & $56(54.9 \%)$ & $27(52.9 \%)$ & $29(56.9 \%)$ \\
\hline Days spent in ICU, mean $\pm S D$ & $17.2 \pm 14.1$ & $20.6 \pm 15.7$ & $|4.1 \pm 1| .8$ \\
\hline Mechanical ventilation, $\mathrm{n}(\%)$ & $38(37.3 \%)$ & $13(25.5 \%)$ & $25(49.0 \%)$ \\
\hline Days spent with mechanical ventilation, mean \pm SD & $13.7 \pm 13.2$ & $15.3 \pm 12.2$ & $12.8 \pm 13.8$ \\
\hline Dialysis, n (\%) & $\mathrm{II}(\mathrm{I} 0.8 \%)$ & $7(13.7 \%)$ & $4(7.8 \%)$ \\
\hline Days spent at the dialysis unit, mean $\pm S D$ & $14.6 \pm 14.2$ & $18.0 \pm 16.7$ & $8.8 \pm 6.6$ \\
\hline \multicolumn{4}{|c|}{ Proportion with tests/procedures supporting IFI diagnosis, n (\%) } \\
\hline Chest radiograph & $86(84.3 \%)$ & $38(74.5 \%)$ & $48(94.1 \%)$ \\
\hline Bronchoalveolar lavage-PCR & $12(11.8 \%)$ & $3(5.9 \%)$ & $9(17.6 \%)$ \\
\hline Galactomannan antigen & $\mathrm{II}(10.8 \%)$ & $2(3.9 \%)$ & $9(17.6 \%)$ \\
\hline
\end{tabular}

Abbreviations: IFI, invasive fungal infection; LOS, length of stay; ER, emergency room; HDU, high dependency unit; ICU, intensive care unit; PCR, polymerase chain reaction; -, data not available. 
Table 4 IFI mortality and 30-day relapse/rehospitalization

\begin{tabular}{|c|c|c|c|}
\hline Outcome & $\begin{array}{l}\text { Overall } \\
(\mathrm{N}=102)\end{array}$ & $\begin{array}{l}\text { Saudi Arabia } \\
(n=5 I)\end{array}$ & $\begin{array}{l}\text { Lebanon } \\
(n=51)\end{array}$ \\
\hline In-hospital mortality, \% & 42 & 35 & 49 \\
\hline \multicolumn{4}{|l|}{ Cause of death ${ }^{\mathrm{a}}$} \\
\hline $\begin{array}{l}\text { Underlying disease-related } \\
\text { complication, \% }\end{array}$ & 72 & 94 & 56 \\
\hline IFI related, \% & 33 & 6 & 52 \\
\hline Other, \% & 7 & 11 & 4 \\
\hline \multicolumn{4}{|l|}{$\begin{array}{l}\text { Outcomes } 30 \text { days post } \\
\text { discharge }^{b}\end{array}$} \\
\hline Relapse of IFI, \% & 2 & 3 & 0 \\
\hline Rehospitalization for IFI, \% & 2 & 3 & 0 \\
\hline $\begin{array}{l}\text { Rehospitalization, any } \\
\text { reason, \% }\end{array}$ & 14 & 9 & 19 \\
\hline
\end{tabular}

Notes: Multiple causes of death could be listed. 'Only measured in patients discharged alive ( $n=59$ overall, 33 from Saudi Arabia and 26 from Lebanon).

Abbreviation: IFI, invasive fungal infection.

attributable to resource utilization, such as hospitalization, would be expected to vary across countries due to differences in practices and health care systems.

This study has a number of potential limitations associated with retrospective research, including representativeness beyond the countries involved in data collection and selection bias. We were unable to attribute health care resource utilization that occurred specifically due to IFI versus for underlying conditions within the patient; as such, resource utilization represents an all-cause, not disease-specific, utilization among IFI patients. Nevertheless, this study represents the first comprehensive attempt to benchmark the real-world clinical and economic burden of IFI in countries within the Middle East. Research in other countries would be needed to confirm the findings reported here.

\section{Conclusion}

This real-world observational study demonstrates that many patients from the sites in Lebanon and Saudi Arabia who developed IFI included hosts with a variety of comorbidities that may put them at risk for fungal infections. Clinical and economic outcomes in this population demonstrate significant burden with high mortality risk and long hospitalizations and suboptimal use of antifungal agents. The results of this study may encourage use of clinical guidelines to treat IFI in the Middle East.

\section{Acknowledgment}

This study was sponsored by Pfizer. The authors would like to thank Monica Katyal for her assistance with data collection and analysis.

\section{Author contributions}

Adel F Alothman, Abdulhakeem O Althaqafi, Madonna J Matar, Rima Moghnieh, Nirvana Raghubir, Claudie Charbonneau, Caitlyn T Solem, and Jennifer M Stephens contributed to study design. Cynthia Macahilig, Adel F Alothman, Abdulhakeem O Althaqafi, Madonna J Matar, Rima Moghnieh, Thamer H Alenazi, and Fayssal M Farahat were involved in data acquisition. Caitlyn T Solem and Shelby Corman undertook data analysis. All authors contributed to data interpretation, manuscript drafting, and approved the final manuscript. All authors contributed toward data analysis, drafting and critically revising the paper and agree to be accountable for all aspects of the work.

\section{Disclosure}

Shelby Corman, Jennifer M Stephens, and Caitlyn T Solem are employees of Pharmerit International, who were paid consultants to Pfizer in connection with the development of this manuscript and study design, management, and statistical analysis for this study. Cynthia Macahilig is an employee of Medical Data Analytics, a subcontractor to Pharmerit International, who was a paid consultant to Pfizer for the study design, management, and data collection for this study. Nirvana Raghubir and Claudie Charbonneau are employees and shareholders of Pfizer. Adel F Alothman received honoraria for several presentations from Pfizer, MSD, and Al-Hikmah University; travel support from Pfizer, MSD, Gilead Sciences, and Al-Hikmah University to attend symposia; and honoraria for patient data collection related to this study from Pfizer. Abdulhakeem O Althaqafi received a research grant (RR13/248/J) sponsored by Pfizer and received honoraria for patient data collection related to this study from Pfizer. Fayssal M Farahat received honoraria for patient data collection related to this study from Pfizer. Madonna J Matar has received travel support for attending meetings and received honoraria for patient data collection related to this study from Pfizer. Rima Moghnieh received honoraria for patient data collection related to this study from Pfizer and sponsorship for attending medical meetings from Pfizer and MSD. Thamer H Alenazi received travel support for attending medical meetings from MSD and Pfizer. The authors report no other conflicts of interest in this work.

\section{References}

1. Al-Abdely HM, Alothman AF, Salman JA, et al. Clinical practice guidelines for the treatment of invasive Aspergillus infections in adults in the Middle East region: expert panel recommendations. J Infect Public Health. 2014;7(1):20-31. 
2. Alothman AF, Al-Musawi T, Al-Abdely HM, et al. Clinical practice guidelines for the management of invasive Candida infections in adults in the Middle East region: expert panel recommendations. J Infect Public Health. 2014;7(1):6-19.

3. Morrell M, Fraser VJ, Kollef MH. Delaying the empiric treatment of Candida bloodstream infection until positive blood culture results are obtained: a potential risk factor for hospital mortality. Antimicrob Agents Chemother. 2005;49(9):3640-3645.

4. Pfaller MA, Jones RN, Doern GV, Sader HS, Hollis RJ, Messer SA. International surveillance of bloodstream infections due to Candida species: frequency of occurrence and antifungal susceptibilities of isolates collected in 1997 in the United States, Canada, and South America for the SENTRY program. The SENTRY Participant Group. J Clin Microbiol. 1998;36(7):1886-1889.

5. Parkins MD, Sabuda DM, Elsayed S, Laupland KB. Adequacy of empirical antifungal therapy and effect on outcome among patients with invasive Candida species infections. J Antimicrob Chemother. 2007;60(3):613-618.
6. Wey SB, Mori M, Pfaller MA, Woolson RF, Wenzel RP. Risk factors for hospital-acquired candidemia. A matched case-control study. Arch Intern Med. 1989;149(10):2349-2353.

7. Drgona L, Khachatryan A, Stephens J, et al. Clinical and economic burden of invasive fungal diseases in Europe: focus on pre-emptive and empirical treatment of Aspergillus and Candida species. Eur J Clin Microbiol Infect Dis. 2014;33(1):7-21.

8. Baddley JW, Stephens JM, Ji X, Gao X, Schlamm HT, Tarallo M. Aspergillosis in Intensive Care Unit (ICU) patients: epidemiology and economic outcomes. BMC Infect Dis. 2013;13:29.

9. Patel DA, Gao X, Stephens JM, Forshag MS, Tarallo M. US hospital database analysis of invasive Aspergillosis in the chronic obstructive pulmonary disease non-traditional host. J Med Econ. 2011;14(2) $227-237$.
Infection and Drug Resistance

\section{Publish your work in this journal}

Infection and Drug Resistance is an international, peer-reviewed openaccess journal that focuses on the optimal treatment of infection (bacterial, fungal and viral) and the development and institution of preventive strategies to minimize the development and spread of resistance. The journal is specifically concerned with the epidemiology of antibiotic

\section{Dovepress}

resistance and the mechanisms of resistance development and diffusion in both hospitals and the community. The manuscript management system is completely online and includes a very quick and fair peerreview system, which is all easy to use. Visit http://www.dovepress.com/ testimonials.php to read real quotes from published authors. 